Original Contribution

\title{
Activation of c-Jun-N-terminal kinase is required for apoptosis triggered by glutathione disulfide in neuroblastoma cells
}

\author{
Giuseppe Filomeni, Katia Aquilano, Patrizia Civitareale, Giuseppe Rotilio, Maria R. Ciriolo* \\ Department of Biology, University of Rome "Tor Vergata" via della Ricerca Scientifica, 1, 00133 Rome, Italy
}

Received 7 December 2004; revised 18 March 2005; accepted 18 March 2005

Available online 7 April 2005

\begin{abstract}
Changes in intracellular redox status are crucial events that trigger downstream proliferation or death responses through activation of specific signaling pathways. Moreover, cell responses to oxidative challenge may depend on the pattern of redox-sensitive molecular factors. The stress-activated protein kinases c-Jun-N-terminal kinase (JNK) and p38 MAP kinase (p38 ${ }^{\mathrm{MAPK}}$ ) are implicated in different forms of apoptotic neuronal cell death. Here, we investigated the effects, on neuroblastoma cells, of the prooxidant molecule GSSG, which we previously demonstrated to be an efficient proapoptotic compound able to activate the $\mathrm{p} 38^{\mathrm{MAPK}}$ death pathway in promonocytic cells. We found that neuroblastoma cells are not prone to GSSG-induced apoptosis, although the treatment slightly induced growth arrest through the accumulation of $\mathrm{p} 53$ and its downstream target gene, p21. However, GSSG treatment became cytotoxic when cells were previously depleted of intracellular GSH content. Under this condition, apoptosis was triggered by an increased production of superoxide that led to a specific activation of the JNK-dependent pathway. The involvement of superoxide and JNK was demonstrated by cell death inhibition in experiments carried out in the presence of $\mathrm{Cu}, \mathrm{Zn}$ superoxide dismutase or with specific inhibitors of JNK activity. Our data give support to the studies that indicate preferential requirements for the involvement of stress-activated kinases in apoptotic neuronal cells.
\end{abstract}

(c) 2005 Elsevier Inc. All rights reserved.

Keywords: Apoptosis; MAP kinase pathway; Neuroblastoma; Glutathione; ROS; Oxidative stress

\section{Introduction}

Reactive oxygen species (ROS) have been traditionally considered to be damaging to cells. However, a large number of evidence has shown that ROS may also function as important activators of key physiological processes playing a vital role in the cell signaling network [1-3]. The ROS-mediated signaling network relies on a dynamic regulation of the oxidized/reduced form of redox-sensitive

Abbreviations: GSH, reduced glutathione; GSSG, glutathione disulfide; ROS, reactive oxygen species; MAPK, mitogen-activated protein kinase; ASK1, apoptosis signal-regulating kinase; JNK, c-Jun$\mathrm{NH}_{2}$-terminal kinase; $\mathrm{Cu}, \mathrm{Zn} \mathrm{SOD}$, copper, zinc superoxide dismutase; BSO, D,L-buthionine $S, R$-sulfoximine; DCF-DA, 2'-7'-dichlorodihydrofluorescein diacetate; hSOD, SH-SY5Y cells transfected with the human $\mathrm{Cu}, \mathrm{Zn}$ SOD gene.

* Corresponding author. Fax: +3906 72594311

E-mail address: ciriolo@bio.uniroma2.it (M.R. Ciriolo). proteins containing critical cysteines. In this context, glutathione, the most abundant low molecular weight thiol within the cell [4], represents the main buffer system regulating the redox state of these proteins [5].

In the last few years, a direct link between mitogenactivated protein (MAP) kinases signaling network and ROS production has been suggested. In fact, it has been found that redox-sensitive proteins, such as thioredoxin and glutathione $S$-transferase (GST), can directly bind and inactivate apoptosis signal-regulating kinase 1 (ASK1) and JNK1, respectively [6,7]. Under redox unbalance GST and thioredoxin are induced to dissociate, due to the oxidation of specific cysteine residues, thus leading to the activation of their MAP kinase partners. As a result, ASK1 and JNK1 are able to autoactivate and transduce the signal by phospho-activating downstream effectors. This sequence of events represents the switch able to transform a specific redox change (thiol/ disulfide) in a phosphorylative potential that may act in a broad range within the cell. 
Previously, we demonstrated that exogenous GSSG, a membrane-impermeable oxidizing molecule, was able to induce, in U937 promonocytic cells, a rapid alteration of intracellular GSH levels by shifting the equilibrium toward the formation of mixed disulfides with protein thiols [8]. This redox unbalance triggered an apoptotic response by the activation of the canonical mitochondrial pathway, which was preceded by dissociation of the thioredoxin/ASK1 complex and phospho-activation of the p38 MAP kinase (p38 $8^{\mathrm{MAPK}}$ ) pathway. We postulated that such responses could be mediated by specific trans-membrane proteins rich in cysteine residues, which transduce the oxidizing power of GSSG across the plasma membrane through a thiol/disulfide exchange. Since the activation of ASK $1 / \mathrm{p} 38^{\mathrm{MAPK}}$ pathway is also involved in cell response mediated by the engagement of ligands to cysteine-rich death receptors [i.e., those belonging to tumor necrosis factor (TNF) superfamily], we hypothesized that the transduction of oxidizing power could also occur through these trans-membrane proteins. This hypothesis was supported by the evidences that leukemia cells express TNF superfamily-related death receptors [i.e., TNF-related apoptosis-inducing ligand (TRAIL) receptors] at high levels on membrane surfaces, and GSSG was not toxic when added to differentiated macrophages or lymphocytes, although high concentrations were used.

In order to verify whether the cell response to GSSG treatment was hystotype-dependent, we determined the effects of GSSG on neuroblastoma cells. In particular, we found that these cells were resistant to GSSG unless depleted of glutatione content. Focusing on the MAPK signaling pathway activated by GSSG treatment under glutatione deprivation, we found that the predominant MAP kinase pathway to be induced was that mediated by JNK. The results may provide a better understanding about the contribution of cell-specific redox-sensitive proteins in the different cellular responses to oxidative stimuli.

\section{Materials and methods}

\section{Materials}

Oxidized glutathione (GSSG) was from Roche Molecular Biochemicals (Monza, Italy). D,L-Buthionine- $S, R$ sulfoximine (BSO), propidium iodide, protease inhibitor cocktail, IGEPAL CA-630, and anti-p53 (clone BP53-12) and antiactin (clone AC-40) monoclonal antibodies were obtained from Sigma (St. Louis, MO). Anti-p21(clone C-19) polyclonal antibody, monoclonal anti-p-JNK (G-7), and anti p-cJun (KM-1) were from Santa Cruz Biotechnology, Inc. (Santa Cruz, CA). Polyclonal anti-phospho-p38 MAP kinase (Thr180/Tyr182) was from Cell Signaling Technology-New England BioLabs (Beverly, MA). Hoechst 33342, $\mathrm{C}_{5}$-maleimide Alexa Fluor and 2,7'-dichlorofluorescein diacetate (DCF-DA) were from Molecular Probes (Eugene, $\mathrm{OR})$. IgG (H+L)-HRP-conjugated goat anti-mouse and anti- rabbit secondary antibodies were from Bio-Rad Lab. (Hercules, CA). SuperSignal substrate chemiluminescent reagent was from Pierce (Rockford, IL). All other chemicals were obtained from Merck (Darmstadt, Germany).

\section{Cell cultures}

Human neuroblastoma cells SH-SY5Y were purchased from the European Collection of Cell Culture and grown in Dulbecco's modified Eagle's/F12 medium supplemented with $15 \%$ fetal calf serum, at $37^{\circ} \mathrm{C}$ in an atmosphere of $5 \%$ $\mathrm{CO}_{2}$ in air. A monoclonal cell line transfected with human wild-type $\mathrm{Cu}, \mathrm{Zn}$ superoxide dismutase (hSOD cells) was obtained as previously described [9]. Cells were routinely trypsinized and plated at $30 \times 10^{5} / 75-\mathrm{cm}^{2}$ flasks. Cell viability was assessed by trypan blue exclusion.

\section{Treatments}

GSSG solution was prepared by dissolving the purified compound in PBS (phosphate-buffered saline, $10 \mathrm{mM}$ phosphate buffer, $2.7 \mathrm{mM} \mathrm{KCl}, 137 \mathrm{mM} \mathrm{NaCl}, \mathrm{pH}$ 7.4), buffered at $\mathrm{pH} 5.5$ with $\mathrm{NaOH}$, and sterilized by filtration. GSSG was used at concentration of $1 \mathrm{mM}$ at $37^{\circ} \mathrm{C}$ in medium supplemented with serum on the basis of previous results obtained with promonocytic cells [8]. Treatment with BSO was performed at a concentration of $1 \mathrm{mM}$ for $6 \mathrm{~h}$ before the addition of GSSG and maintained in culture media throughout the experiments. Catalase or $\mathrm{Cu}, \mathrm{Zn}$ superoxide dismutase $(\mathrm{Cu}, \mathrm{Zn} \mathrm{SOD})$ were added concomitantly with GSSG and used at a concentration of $1 \mu \mathrm{M}$. Treatments with the cell-permeable JNK inhibitor I and II (SP600125) or p38 ${ }^{\mathrm{MAPK}}$ inhibitor SB203580 (Calbiochem, La Jolla, CA) were performed at a concentration of $10 \mu \mathrm{M}$ because lower concentrations did not show significant inhibition and higher concentrations were toxic. All the compounds were added concomitantly with BSO and maintained throughout the experiments.

\section{Detection of intracellular ROS}

For detection of intracellular ROS, cells were incubated with $50 \mu \mathrm{M}$ DCF-DA for $30 \mathrm{~min}$ at $37^{\circ} \mathrm{C}$, scraped, washed, and resuspended in ice-cold PBS. The fluorescent signals derived by reaction of DCF with ROS, of more than 10,000 cells from each sample, were analyzed by recording FL-1 fluorescence by the FACScalibur system. Prior to data collection, propidium iodide was added to the samples for gating out dead cells. Experiments were repeated at least three times with similar results. The data are given as one representative histogram.

\section{Detection of exofacial sulfhydryls}

For detection of external plasma membrane thiols, cells were incubated with $10 \mu \mathrm{M}$ Alexa Fluor $\mathrm{C}_{5}$-maleimide for 
$1 \mathrm{~h}$ at $37^{\circ} \mathrm{C}$. Labeled thiols were visualized by fluorescent microscopy and images of cells were rapidly digitized with a Cool Snap video camera connected to Nikon Eclipse TE200 epifluorescence microscopy. All images were captured under constant exposure time, gain, and offset. Alternatively, cells were scraped, washed, and resuspended in ice-cold PBS and analyzed cytofluorimetrically by recording FL1 fluorescence. The data are given as one representative histogram.

\section{Analysis of cell proliferation and apoptosis}

Cell viability was assessed by performing direct counting of cells upon staining with the vital dye trypan blue using a hemocytometer. Nuclear fragmentation was detected by optical microscopy on slides by vital staining with the DNA-specific cell-permeable dye Hoechst 33342. The fraction of cells with fragmented nuclei among the total cell population was calculated using Hoechst 33342-stained cells, counting at least 300 cells in at least 10 randomselected fields as described [10]. Alternatively, cells were washed with PBS, stained with $50 \mu \mathrm{M}$ propidium iodide solution [11], and analyzed by a FACScalibur system (Becton Dickinson, San Josè, CA).

\section{Western blot analyses}

Cell pellets were resuspended in lysis buffer containing $10 \mathrm{mM}$ Tris-HCl, $\mathrm{pH} 7.4,5 \mathrm{mM}$ EDTA, $150 \mathrm{mM} \mathrm{NaCl}$, $0.5 \%$ IGEPAL CA-630, and protease inhibitor cocktail. After $30 \mathrm{~min}$ incubation on ice, lysates were centrifuged at $22,300 \mathrm{~g}$ for $15 \mathrm{~min}$ at $4^{\circ} \mathrm{C}$ and supernatants were removed and stored at $-80^{\circ} \mathrm{C}$. Protein extracts were loaded on $12 \%$ polyacrylamide gel and transferred onto a nitrocellulose membrane (Millipore, Bedford, MA). Anti-phospho-JNK (1:200), anti-phospho-c-Jun (1:200), anti-p53 (1:5000), antiactin, anti-phospho-p38 (1:1000), and anti-p21 (1:2000) were used as primary antibodies. Appropriate HRP-conjugated secondary antibodies were used to detect immunoreactive bands by incubation with SuperSignal substrate chemiluminescence reagent. Actin was used as loading control. Densitometric analyses were calculated using Quantity One Software (Bio-Rad) and data were normalized with respect to the actin band. Data are reported as arbitrary units with respect to control which was considered equal to 1.00. Immunoblots reported are from one experiment of three that gave similar results.

\section{Glutathione determination}

Intracellular glutathione was assayed upon formation of $S$-carboxymethyl derivatives of free thiols with iodoacetic acid, followed by the conversion of free amino groups to 2,4-dinitrophenyl derivatives by the reaction with 1-fluoro2,4-dinitrobenzene as described by Reed et al. [12]. Cell suspension was washed three times with PBS in order to completely eliminate exogenous GSSG, resuspended, and lysed by repeated cycles of freezing and thawing under liquid nitrogen. Lysates were then divided into two aliquots: one utilized for GSH/GSSG assay, while the other was used to determine mixed disulfides by treating with $300 \mathrm{mM}$ $\mathrm{NaBH}_{4}$ at $40^{\circ} \mathrm{C}$ for 30 min prior to derivatization, as described previously [13]. Data are expressed as nanomoles of GSH or GSSG per milligram protein. Proteins were determined by the method of Lowry et al. [14].

\section{Data presentation}

All experiments were done with $n=6$ unless otherwise stated. Each assay was performed at least in triplicate under identical conditions. The results are presented as means \pm SD. Statistical evaluation was conducted by ANOVA, followed by correction with Bonferroni's. Comparisons were considered to be significant at $P<0.05$.

\section{Results}

GSSG treatment does not induce apoptosis in SH-SY5Y neuroblastoma cells

We previously demonstrated that externally added GSSG acts as a prooxidant and proapoptotic molecule in human promonocytic U937 cells [8]. The first step of the present study was to verify whether GSSG-mediated toxicity could represent a widespread phenomenon, independent on the cell type. To this end, the dose of $1 \mathrm{mM} \mathrm{GSSG}$, that was successful in inducing apoptosis in promonocytic cells, was supplied to SH-SY5Y neuroblastoma cells. Cell cycle analyses, as well as routine morphological examination, revealed that SH-SY5Y cells were resistant to GSSGinduced apoptosis. In fact, counting of direct viable cells evidenced only a slight inhibition of cell growth after $48 \mathrm{~h}$ of GSSG administration (Fig. 1A). This phenomenon was paralleled by a slight increase in the expression level of cell cycle regulators $\mathrm{p} 53$ and $\mathrm{p} 21$, as demonstrated by Western blot analyses of total cell lysates (Fig. 1B). Moreover, these events were not associated with induction of cell death, as the number of fragmented nuclei after DNA-specific dye with Hoechst 33342 (marker of apoptosis) or lactic dehydrogenase activity in culture media (marker of necrosis) were not significantly different from untreated cells (data not shown).

To further elucidate the GSSG inefficacy on neuroblastoma cells, we tested whether GSSG could affect the thiol redox state of plasma membrane proteins and/or intracellular compartment of this cell line. To this aim, cells were labeled with $10 \mu \mathrm{M}$ Alexa Fluor $\mathrm{C}_{5}$-maleimide, a specific nonpermeable thiol-reactive compound able to recognize and covalently bind to membrane surface cysteines. Fig. 2A shows that $24 \mathrm{~h}$ treatment with $1 \mathrm{mM}$ GSSG had no remarkable effect on extracellular thiol 


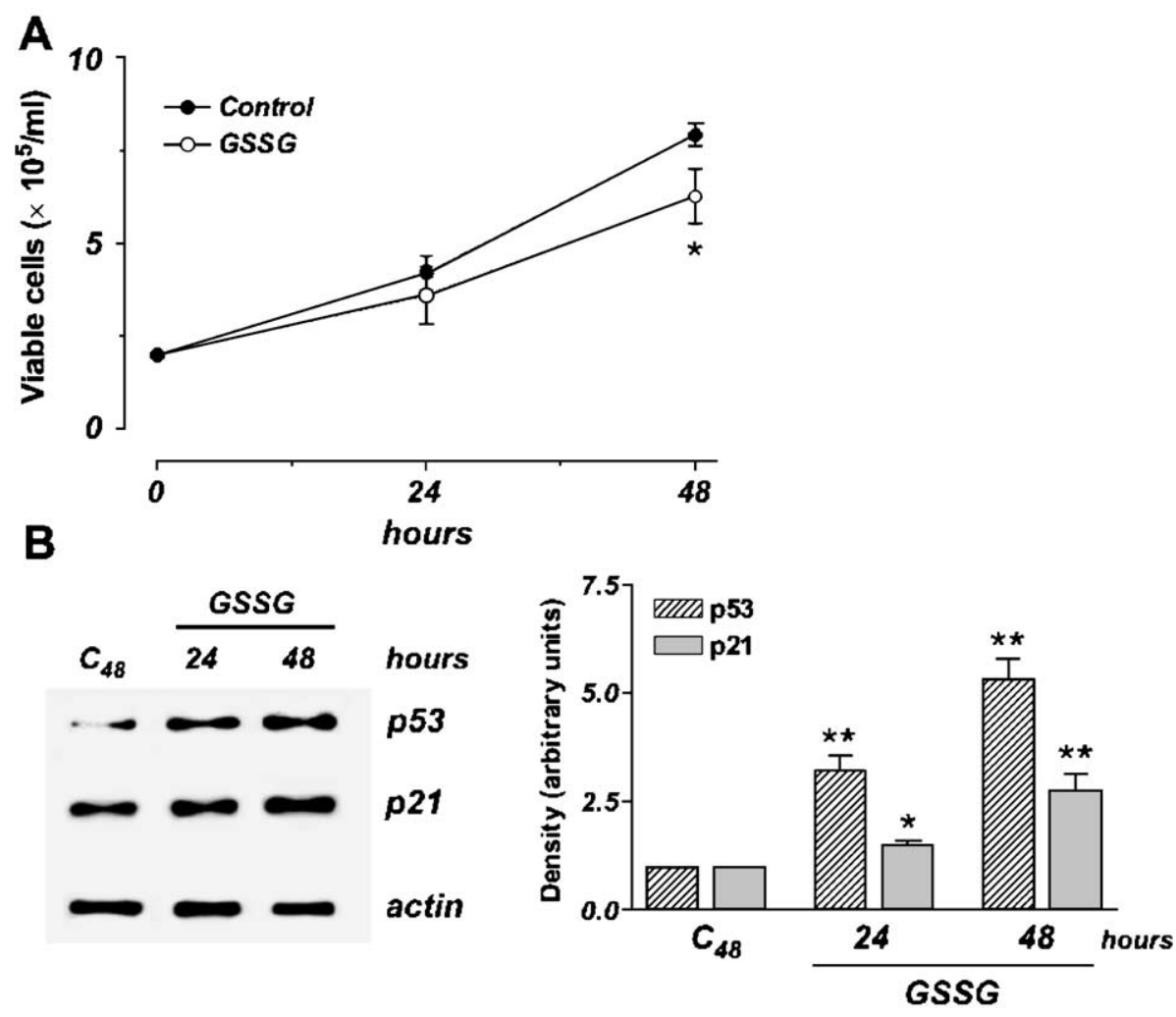

Fig. 1. GSSG inhibits cell growth in SH-SY5Y cells. (A) SH-SY5Y cells were treated with 1 mM GSSG. At indicated time points, cells were counted upon trypan blue staining. Data are presented as means $\pm \mathrm{SD}(n=6) . * P<0.01$ with respect to untreated cells. (B) SH-SY5Y cells were treated with 1 mM GSSG. At indicated time points, p53 and p21 protein levels were determined on total protein extracts $(50 \mu \mathrm{g})$ by Western blot analyses. Actin was used as loading control. The immunoblots shown are from one experiment representative of three that gave similar results. Densitometric analyses of each lane were calculated using Quantity One Software and data are expressed as arbitrary units as described under Materials and methods. Data are presented as means \pm SD. $* * P<0.001 ; * P<$ 0.01 with respect to untreated cells.

content. Cytofluorimetric analyses were also performed and, as shown in Fig. 2B, only a slight decrease in the green fluorescence of $\mathrm{C}_{5}$ maleimide was detected after GSSG treatment, confirming that it was not able to efficiently oxidize extracellular cysteines residues of membrane proteins, when compared with the effects that we observed in U937 cells [8]. The ineffectiveness of GSSG treatment on surface cysteines was mirrored by an almost unchanged intracellular redox state. Indeed, only a mild alteration in intracellular GSH levels was observed after $48 \mathrm{~h}$ of treatment with $1 \mathrm{mM}$ GSSG, due to its engagement in mixed disulfide formation with protein thiols (GS-R) (Fig. 2C). Moreover, intracellular GSSG levels paralleled the decrease of GSH content, keeping the ratio GSH/GSSG unchanged, also confirming that externally added GSSG was not able to cross the plasma membrane (Fig. 2C).

\section{Depletion of intracellular GSH sensitizes SH-SY5Y cells to GSSG treatment}

To determine whether the significant intracellular decrement of GSH, which was the mediator of exogenous GSSG cytotoxicity in U937, could be also effective in neuroblastoma cells, we lowered the tripeptide content of $\mathrm{SH}$ -
SY5Y cells by treatment with $1 \mathrm{mM}$ BSO, a specific inhibitor of GSH synthesis. Fig. 3 shows that BSO treatment caused a time-dependent decrease of both GSH and GSSG that was not altered by the treatment with GSSG (data not shown). Furthermore BSO treatment restored cell sensitivity to GSSG; in particular, we observed, by cytofluorimetric analyses after staining with propidium iodide that SH-SY5Y cells treated with BSO alone are committed to apoptosis to a low extent (12\% after $48 \mathrm{~h})$. This effect was dramatically increased with $1 \mathrm{mM}$ GSSG treatment in a time-dependent manner (49 and 65\% at 24 and 48 h, respectively) (Fig. 3B). Apoptosis was associated with a strong increase in the expression levels of p21 and p53 (Fig. 3C). Moreover, the p53-immunoreactive band was shifted to a higher molecular weight, which corresponds to the phosphorylated form of the protein [15], indicating a stronger activation of the transcription factor under this condition (Fig. 3C).

GSSG treatment induces the activation of JNK in GSH-depleted SH-SY5Y cells

Several studies report that under ROS challenge neuronal cells are preferentially committed to apoptotic cell death by the activation of the MAP kinase signaling pathway [16, 
A
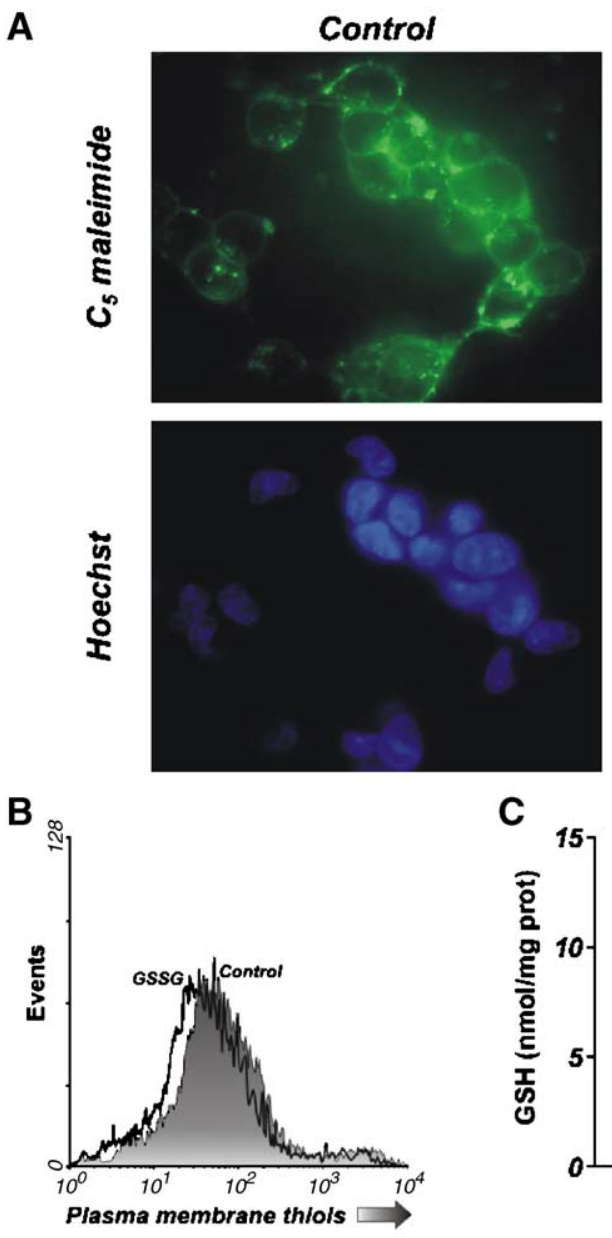

C
GSSG 24h
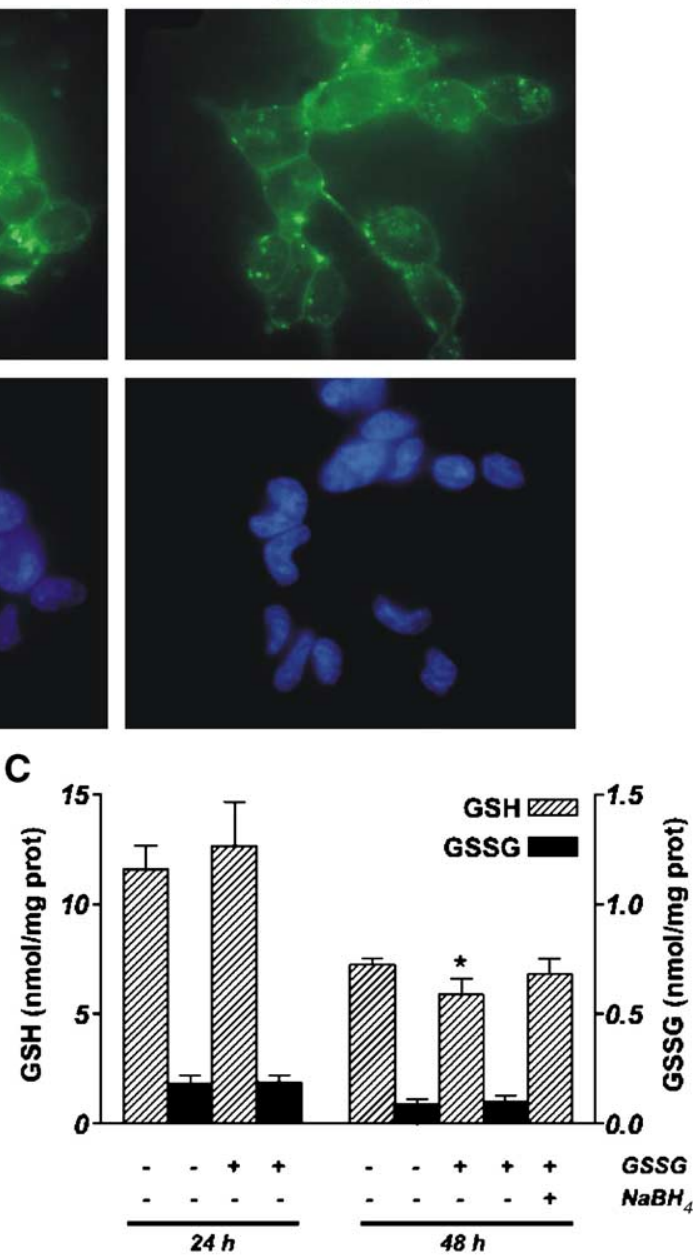

Fig. 2. GSSG slightly affects exofacial membrane thiols and the intracellular glutathione redox state. (A) SH-SY5Y cells were treated with $1 \mathrm{mM}$ GSSG. After $24 \mathrm{~h}$, cells were analyzed by fluorescent microscopy after incubation with $10 \mu \mathrm{M}$ Alexa Fluor $\mathrm{C}_{5}$-maleimide to visualize membrane thiols and Hoechst 33342 to stain nuclei. (B) Alternatively, Alexa Fluor $\mathrm{C}_{5}$-maleimide-stained cells were analyzed cytofluorimetrically by FACScalibur system. The histograms reported are from one experiment representative of three that gave similar results. (C) SH-SY5Y cells were treated with $1 \mathrm{mM} \mathrm{GSSG}$. At indicated time points, cells were used for GSH and GSSG determinations by HPLC. $\mathrm{NaBH}_{4}$ treatment was used to reduce mixed disulfides (see Materials and methods). Data are expressed as means $\pm \mathrm{SD}(n=6) .{ }^{*} P<0.01$ with respect to untreated cells.

17]. To establish whether the effect of GSSG on SH-SY5Ydepleted cells was mediated by MAP kinases activation, we analyzed the expression levels of the phospho-activated forms of JNK and $\mathrm{p} 38^{\mathrm{MAPK}}$. As seen in Fig. 4A, BSO treatment alone was able to efficiently induce the activation of $\mathrm{p} 38^{\mathrm{MAPK}}$ (P-p38 $8^{\mathrm{MAPK}}$ ) confirming that this kinase is extremely sensitive to changes of GSH content. Conversely, the combined treatment of BSO with GSSG induced a timedependent decrease in the intensity of the P-p38 $8^{\mathrm{MAPK}}$ immunoreactive band from 6 to $24 \mathrm{~h}$, and a significant activation of JNK (P-JNK) and its downstream target c-Jun (P-c-Jun). In order to confirm the predominant role of JNK in the induction of apoptosis by GSSG in GSH-depleted cells, we performed experiments in the presence of inhibitors of both kinases. Fig. 4B shows cytofluorimetric analyses of apoptotic cells after staining with propidium iodide. Pretreatment with JNK inhibitors I and II
(SP600125) had no effect on BSO-treated SH-SY5Y, but strongly reduced apoptosis in GSH-depleted cells after $24 \mathrm{~h}$ incubation with GSSG $(-68 \%)$. On the other hand, the p3 $8^{\mathrm{MAPK}}$ inhibitor, SB203580, completely reverted the even low extent of BSO-induced apoptosis, and significantly reduced the percentage of apoptotic cells after cotreatment $\mathrm{BSO} / \mathrm{GSSG}(-12 \%)$.

GSSG induces apoptosis in GSH-depleted SH-SY5Y cells through superoxide production

In searching for the molecular mechanisms that are responsible for the induction of apoptosis under GSH depletion, we considered that $\gamma$-glutamyl transpeptidase, the main enzyme involved in the Meister cycle able to catalyze the cleavage of GSH-deriving $\gamma$-glutamate, is also able, but with a much lower efficiency, to catalyze the 

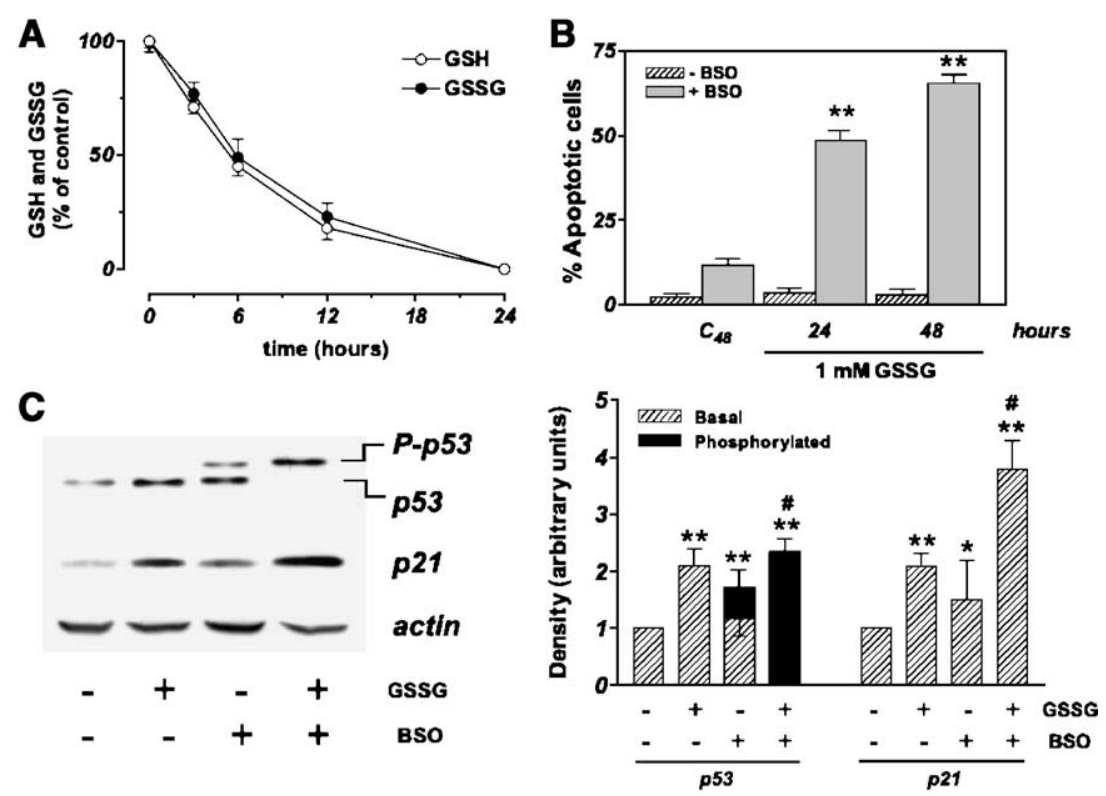

Fig. 3. GSSG induces apoptotic cell death in GSH-depleted SH-SY5Y cells. (A) SH-SY5Y cells were treated with 1 mM BSO. At the indicated time points cells were used for GSH and GSSG determinations by HPLC. Data are expressed as means \pm SD $(n=4)$ and are all significantly different with respect to untreated cells. (B) SH-SY5Y cells were pretreated with $1 \mathrm{mM} \mathrm{BSO}$ for $6 \mathrm{~h}$ before the addition of $1 \mathrm{mM} \mathrm{GSSG}$ and maintained in culture medium throughout the experiment. At 24 or $48 \mathrm{~h}$ after GSSG addition, apoptotic cells were determined by the FACScalibur system after staining with propidium iodide. Data are expressed as means $\pm \mathrm{SD}(n=6)$. ${ }^{* *} P<0.001$ with respect to BSO-treated cells. (C) SH-SY5Y cells were pretreated with $1 \mathrm{mM}$ BSO for $6 \mathrm{~h}$ before the addition of $1 \mathrm{mM} \mathrm{GSSG}$ and maintained in culture medium throughout the experiment. p53 and p21 protein levels were determined, at $24 \mathrm{~h}$ after GSSG addition, on total protein extracts $(50 \mu \mathrm{g})$ by Western blot analyses. Actin was used as loading control. The immunoblots shown are from one experiment representative of three that gave similar results. Densitometric analyses of each lane were calculated using Quantity One Software and data are expressed as arbitrary units as described under Materials and methods. Data are presented as means $\pm \mathrm{SD}$. ${ }^{*} P<0.001 ; * P<0.01$ with respect to untreated cells; $\# P<0.001$ with respect to BSO- and GSSG-treated cells. P-p53: phospho-p53.

cleavage of GSSG, thus producing $\mathrm{H}_{2} \mathrm{O}_{2}$ at the level of plasma membrane [18]. In this way, BSO treatment, by decreasing GSH content, could determine a higher sensitivity of SH-SY5Y cells to $\mathrm{H}_{2} \mathrm{O}_{2}$-mediated oxidative stress, with this event the real inducer of cell death. Moreover, GSSG can also produce superoxide anions $\left(\mathrm{O}_{2}{ }^{-}\right)$from the reaction between $\mathrm{O}_{2}$ and thiyl radicals. To check these assumptions, SH-SY5Y GSH-depleted cells were incubated with DCF-DA and assayed for ROS production through cytofluorimetric analyses. Fig. 5A shows that, although ROS production was slightly influenced by BSO treatment, the percentage of increase observed in combination with GSSG treatment was significantly higher compared with untreated cells.

In order to identify the ROS species involved $\left(\mathrm{O}_{2}{ }^{{ }^{-}}\right.$or $\mathrm{H}_{2} \mathrm{O}_{2}$ ), we performed experiments in the presence of ROS scavengers. Fig. 5B shows that, even in the presence of 1 $\mu \mathrm{M}$ catalase in culture medium, SH-SY5Y underwent apoptosis to the same extent as control cells. Conversely, by augmenting $\mathrm{O}_{2}{ }^{--}$elimination either by exogenously adding $\mathrm{Cu}, \mathrm{Zn}$ SOD $(1 \mu \mathrm{M})$ or by endogenously increasing its expression (hSOD cells), we were able to significantly decrease apoptotic cell death by $50 \%$, implying that $\mathrm{O}_{2}{ }^{-}$ was the radical specie involved in the activation process (Fig. 5B). The protective role exerted by $\mathrm{Cu}, \mathrm{Zn}$ SOD was due to the efficient removal of radicals as shown by the assay of ROS with DCF-DA (Fig. 5A).

\section{Discussion}

Previously we demonstrated that promonocytic U937 cells, treated with GSSG, were committed to cell death by apoptosis through the activation of the stress-activated protein kinase, $\mathrm{p} 38^{\mathrm{MAPK}}$. Since, owing to its hydrophilic nature GSSG is unable to cross the cell membrane, we have hypothesized that its effects inside the cell could occur through a putative thiol/disulfide exchange relay along cysteine residues of proteins spanning across the plasma membrane. The external cysteine residues were able to transduce the prooxidant power intracellularly by shifting the GSH content toward the production of mixed disulfides. The resulting redox imbalance was responsible for $\mathrm{p} 38^{\mathrm{MAPK}}$ activation and cell death induction [8]. Here we demonstrate that this pathway is not of general application because neuroblastoma cells are resistant to GSSG treatment. The major divergence between the two cell lines was that GSSG does not efficiently oxidize exofacial cysteine thiols in neuroblastoma cells, although they are present at high concentrations. This points out that the redox potential of cysteine residues could be different, depending on the protein species; however, we cannot exclude that critical cysteines on specific membrane proteins could be modulated under those conditions. As a consequence of the unreactivity of membrane cysteines, the oxidizing power of GSSG was not efficiently transduced intracellularly, as the 
A
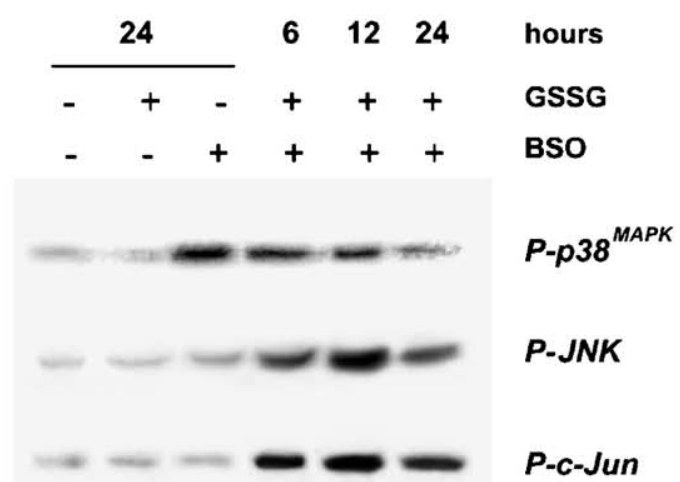

\section{P-JNK}

\section{P-c-Jun}

actin

\begin{tabular}{|c|c|c|c|c|c|c|}
\hline hours & \multicolumn{3}{|c|}{24} & 6 & 12 & 24 \\
\hline GSSG & - & + & - & + & + & + \\
\hline BSO & - & - & + & + & + & + \\
\hline P-p38 ${ }^{\text {MAPK }}$ & 1.00 & $0.78 \pm 0.42$ & $4.83 \pm 0.28^{\star \star}$ & $3.93 \pm 0.21^{\star \star}$ & $3.04 \pm 0.46^{\star \star}$ & $1.63 \pm 0.86$ \\
\hline P-JNK & 1.00 & $1.34 \pm 0.21$ & $1.71 \pm 0.66$ & $7.58 \pm 0.49^{\star \star}$ & $11.40 \pm 0.62^{* *}$ & $6.78 \pm 0.41^{\star *}$ \\
\hline P-Jun & 1.00 & $1.11 \pm 0.30$ & $1.03 \pm 0.31$ & $4.82 \pm 0.29^{\star \star}$ & $6.70 \pm 0.46^{\star \star}$ & $5.22 \pm 0.50^{\star \star *}$ \\
\hline
\end{tabular}

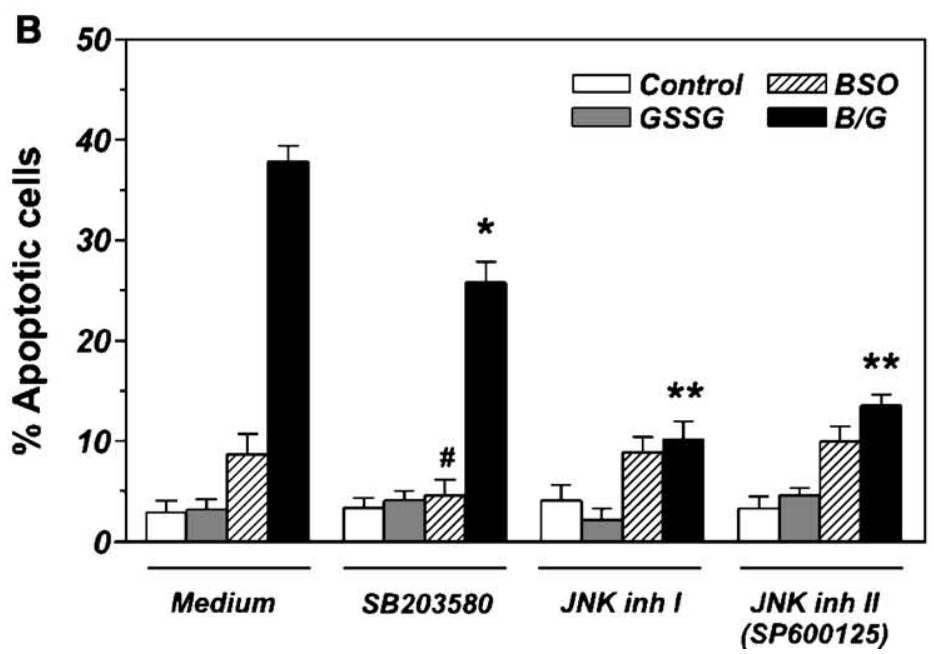

Fig. 4. GSSG-induced apoptosis in GSH-depleted SH-SY5Y cells is associated with the activation of JNK/c-Jun pathway. (A) SH-SY5Y cells were pretreated with $1 \mathrm{mM}$ BSO for $6 \mathrm{~h}$ before the addition of $1 \mathrm{mM} \mathrm{GSSG}$ and maintained throughout the experiment. Phospho-p38 ${ }^{\mathrm{MAPK}}$, phospho-JNK, and phospho-c-Jun protein levels were detected, at indicated time points on total protein extracts $(20 \mu \mathrm{g})$ by Western blot analyses. Actin was used as loading control. The immunoblots shown are from one experiment representative of three that gave similar results. $P$-p 38 , phospho-p $38^{\mathrm{MAPK}} ;{ }$-JNK, phospho-JNK; $P$-c-Jun, phospho-c-Jun. Densitometric analyses, shown in the table at the bottom, are expressed as arbitrary units as described under Materials and methods. Data are presented as means $\pm \mathrm{SD}$. ${ }^{* *} P<0.001$ with respect to untreated cells. (B) SH-SY5Y cells were pretreated with $1 \mathrm{mM}$ BSO for $6 \mathrm{~h}$ before the addition of $1 \mathrm{mM}$ GSSG and maintained in culture medium throughout the experiment. JNK and p38 ${ }^{\mathrm{MAPK}}$ inhibitors were added concomitantly with BSO and maintained throughout the experiments. Twenty-four hours after GSSG addition, apoptotic cells were determined by the FACScalibur system after staining with propidium iodide. JNK inh I, JNK inhibitor I; JNK inh II, JNK inhibitor II (or SP600125); SB203580,p38 ${ }^{\mathrm{MAPK}}$ inhibitor. Data are presented as means $\pm \mathrm{SD}(n=4)$. $* * P<$ $0.001 ; * P<0.01$ with respect to $\mathrm{B} / \mathrm{G}$-treated cells; $\# P<0.01$ with respect to BSO-treated cells. 

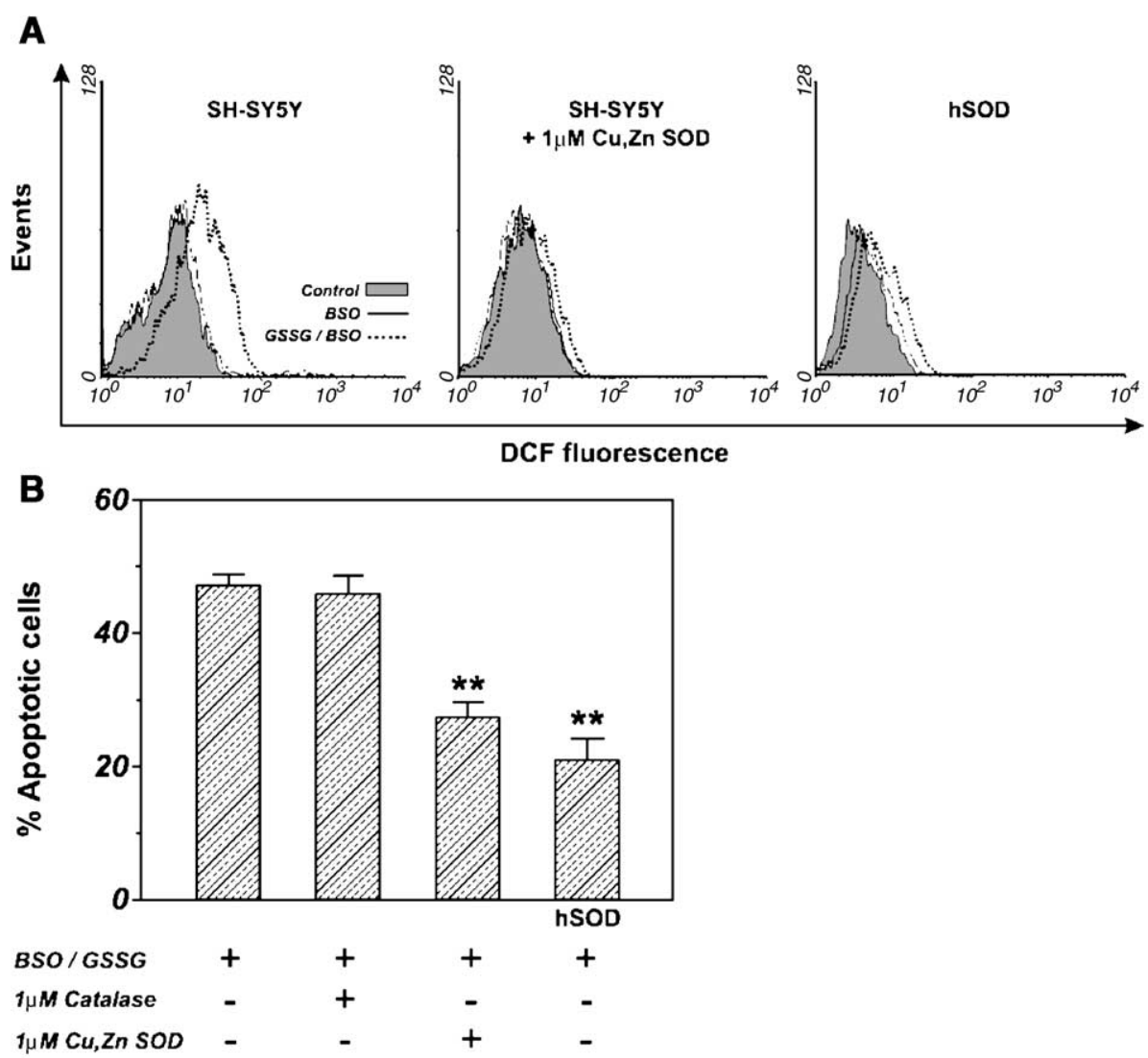

Fig. 5. GSSG induces apoptotic cell death in GSH-depleted SH-SY5Y cells through production of superoxide. (A) Cells were pretreated with $1 \mathrm{mM}$ BSO for $6 \mathrm{~h}$ before the addition of $1 \mathrm{mM} \mathrm{GSSG}$ and maintained in culture medium throughout the experiment. Thirty minutes before the indicated time points, cells were stained with DCF-DA and ROS concentration was detected by FACScalibur system. The histograms reported are from one experiment representative of three that gave similar results. $h S O D$, SH-SY5Y cells overexpressing human Cu,Zn SOD. (B) SH-SY5Y cells were pretreated with $1 \mathrm{mM}$ BSO for $6 \mathrm{~h}$ before the addition of $1 \mathrm{mM}$ GSSG and maintained in culture medium throughout the experiment. $1 \mu \mathrm{M}$ catalase or $1 \mu \mathrm{M} \mathrm{Cu}, \mathrm{Zn}$ SOD was added concomitantly with GSSG. Twenty-four hours after GSSG addition, apoptotic cells were determined by the FACScalibur system after staining with propidium iodide. Data are presented as means $\pm \mathrm{SD}(n=4) .{ }^{* *} P<0.001$ with respect to BSO/GSSG-treated cells.

GSH content was slightly decreased only after $48 \mathrm{~h}$ of treatment. The mild oxidative unbalance occurring in neuroblastoma cells upon treatment with GSSG was not sufficient to switch on oxidative-dependent apoptotic program(s), but cells only slightly arrested their growth as demonstrated by the accumulation of p53 and of its downstream activated gene $\mathrm{p} 21$.

However, GSSG was cytotoxic in neuroblastoma cells when the intracellular content of GSH was lowered by BSO; in fact, SH-SY5Y, depleted of GSH, loose their resistance to GSSG insult and efficiently undergo apoptosis. In the search for the molecular mechanisms underlying the sensitivity to GSSG under this condition, we took into account the opportunity for GSSG to transiently generate the thiyl radical which could efficiently react with oxygen, thus producing $\mathrm{O}_{2}{ }^{--}$. Under basal conditions, due to the low extent of reaction between GSSG and protein thiols, $\mathrm{O}_{2}{ }^{-}$can be efficiently scavenged by cellular antioxidant equipment, including GSH. On the contrary, in GSH-depleted cells, $\mathrm{O}_{2}{ }^{--}$may become the inducer of the apoptotic process triggered by GSSG. This was nicely demonstrated by the experiments carried out in the presence of enzymatic ROS scavengers. Cell death was efficiently inhibited only by the addition of $\mathrm{Cu}, \mathrm{Zn}$ SOD to the culture medium of GSHdepleted cells or by using SH-SY5Y cells overexpressing $\mathrm{Cu}, \mathrm{Zn}$ SOD (Fig. 6). These results, while in agreement with the role played by this enzyme in modulating the sensitivity of cancer cells to external or cytosolic-derived superoxide [19], may also be in line with the ineffectiveness of $\mathrm{Cu}, \mathrm{Zn}$ SOD to counteract mitochondrial-mediated ROS production [20].

After oxidative stimuli, such as UV or hydrogen peroxide, several members of the MAP kinase family have been described to be phospho-activated on specific ser/thr residues $[17,21]$. This modification leads to the activation of numerous downstream transcription factors which mediate the cell response. We propose the activation of two distinct apoptotic pathways in GSH-depleted cells. The first one is mediated by $\mathrm{p} 38^{\mathrm{MAPK}}$ and seems to be only responsive to BSO pretreatment: the level of phospho-p $38^{\mathrm{MAPK}}$ was increased in GSH-depleted cells and its inhibition completely prevented BSO-induced apoptosis (about 10\%). The second one, which represents the main apoptotic pathway, is mediated by JNK. To confirm this scheme, the percentage of 


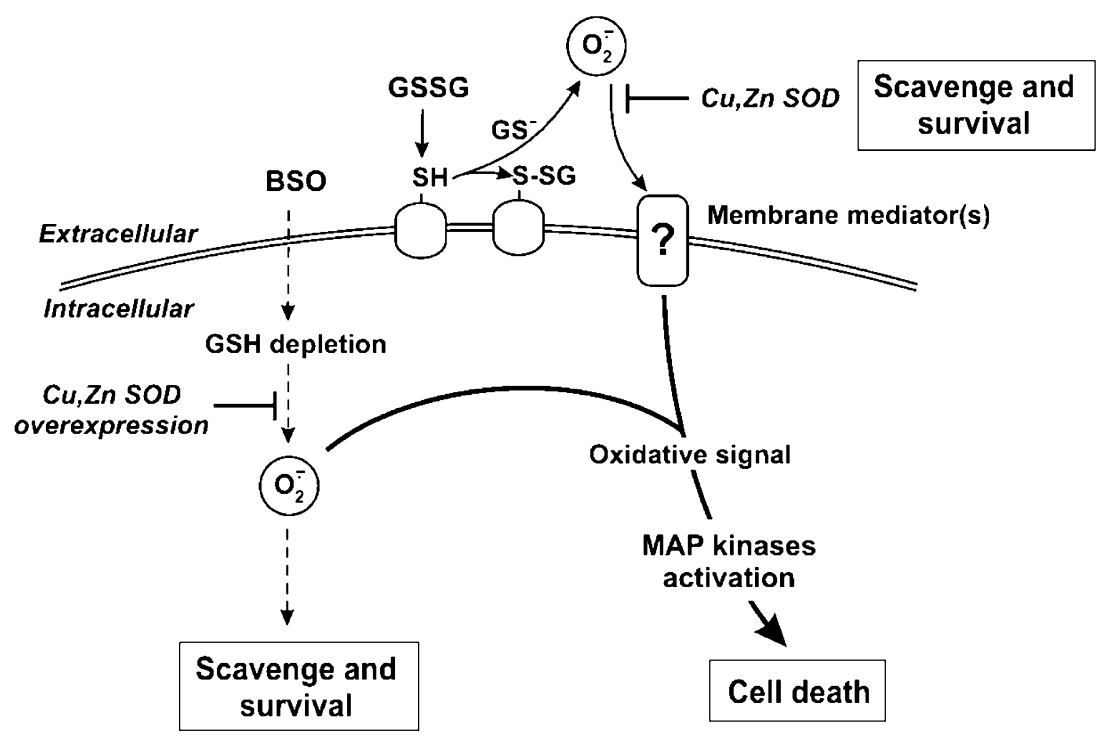

Fig. 6. Shematic diagram of the effects of GSSG on GSH-depleted SH-SY5Y cells.

cells rescued from apoptosis upon JNK inhibitors addition accounts for the majority of apoptotic cells (about 26\%) and it was additional to that obtained upon treatment with BSO alone. Moreover, the appearance of a phospho-JNK-immunoreactive band becomes significantly evident as early as $6 \mathrm{~h}$ of treatment remaining highly expressed all during the experimental time. Therefore, these results strongly support our hypothesis [8] that $\mathrm{p} 38^{\mathrm{MAPK}}$ represents a first sensor of the GSH redox state, while JNK activation could represent the response of GSH-depleted cells to $\mathrm{O}_{2}{ }^{--}$production after treatment with GSSG. The results, while confirming distinct requirements for the induction of the two MAP kinases in different forms of neuronal apoptotic death [22], at the same time need further studies to elucidate the down-regulation/ inhibition of $\mathrm{p} 38^{\mathrm{MAPK}}$ observed upon JNK activation. Our results are also in agreement with several observations suggesting that JNK is involved in neuronal cell death during early brain development [23], in neurodegenerative disorders [24] or under oxidative insult [25].

Overall the results demonstrate that GSSG may behave as a proapoptotic agent by two distinct mechanisms depending on the cell type: one is mediated by direct thiol/disulfide exchange, the other by the formation of $\mathrm{O}_{2}{ }^{-}$. The two mechanisms, in turn, involve two different apoptotic pathways mediated by specific MAP kinase members. This different behavior of different cell types may also provide a clue for a valuable therapeutic approach. The specific apoptotic effect exerted by GSSG on U937 could be applied in chemotherapy in order to selectively kill leukemia-derived cells without compromising the viability of other cells, especially in the case of acquired resistance to the conventional anticancer drugs. Moreover, a use of GSSG in combination with other molecules, able to decrease intracellular GSH content, could be proposed for cancer cells resistant to the GSSG treatment alone.
Work is in progress in our laboratory in order to identify the cell-specific cysteine-rich proteins able to sense GSSGmediated oxidative stimulus and to transduce it in an efficient apoptotic signal within the cell. This could be very important in order to identify new molecular targets for GSSG and to propose a selective use of this compound in cancer therapy.

\section{Acknowledgments}

This work was partially supported by "Special Projects" from FIRB, MIUR, CNR, and Ministero della Salute.

\section{References}

[1] Adler, V.; Yin, Z.; Tew, K. D.; Ronai, Z. Role of redox potential and reactive oxygen species in stress signaling. Oncogene 18: 6104-6111; 1999.

[2] Finkel, T. Reactive oxygen species and signal transduction. IUBMB Life 52:3-6; 2001.

[3] Forman, H. J.; Torres, M.; Fukuto, J. Redox signaling. Mol. Cell. Biochem. 234-235:49-62; 2002.

[4] Filomeni, G.; Rotilio, G.; Ciriolo, M. R. Cell signalling and the glutathione redox system. Biochem. Pharmacol. 64:1057-1064; 2002.

[5] Schafer, F. Q.; Buettner, G. R. Redox environment of the cell as viewed through the redox state of the glutathione disulfide/glutathione couple. Free Radic. Biol. Med. 30:1191-1212; 2001.

[6] Saitoh, M.; Nishitoh, H.; Fujii, M.; Takeda, K.; Tobiume, K.; Sawada, Y.; Kawabata, M.; Miyazono, K.; Ichijo, H. Mammalian thioredoxin is a direct inhibitor of apoptosis signal-regulating kinase (ASK) 1. EMBO J. 17:2596-2606; 1998.

[7] Adler, V.; Yin, Z.; Fuchs, S. Y.; Benezra, M.; Rosario, L.; Tew, K. D.; Pincus, M. R.; Sardana, M.; Henderson, C. J.; Wolf, C. R.; Davis, R. J.; Ronai, Z. Regulation of JNK signaling by GSTp. EMBO J. 18:1321-1334; 1999.

[8] Filomeni, G.; Rotilio, G.; Ciriolo, M. R. Glutathione disulfide 
induces apoptosis in U937 cells by a redox-mediated p38 MAP kinase pathway. FASEB J. 17:64-66; 2003.

[9] Carrì, M. T.; Ferri, A.; Battistoni, A.; Famhy, L.; Gabbianelli, R.; Poccia, F.; Rotilio, G. Expression of a $\mathrm{Cu}, \mathrm{Zn}$ superoxide dismutase typical of familial amyotrophic lateral sclerosis induces mitochondrial alteration and increase of cytosolic $\mathrm{Ca} 2+$ concentration in transfected neuroblastoma SH-SY5Y cells. FEBS Lett. 414:365-368; 1997.

[10] Ghibelli, L.; Fanelli, C.; Rotilio, G.; Lafavia, E.; Coppola, S.; Colussi, C.; Civitareale, P.; Ciriolo, M. R. Rescue of cells from apoptosis by inhibition of active GSH extrusion. FASEB J. 12: 479-486; 1998

[11] Nicoletti, I.; Migliorati, G.; Pagliacci, M. C.; Grignani, F.; Riccardi, C. A rapid and simple method for measuring thymocyte apoptosis by propidium iodide staining and flow cytometry. J. Immunol. Methods 139:271-279; 1991

[12] Reed, D. J.; Babson, J. R.; Beatty, P. W.; Brodie, A. E.; Ellis, W. W.; Potter, D. W. High-performance liquid chromatography analysis of nanomole levels of glutathione, glutathione disulfide, and related thiols and disulfides. Anal. Biochem. 106:55-62; 1980.

[13] Ciriolo, M. R.; Palamara, A. T.; Incerpi, S.; Lafavia, E.; Bue, M. C.; De Vito, P.; Garaci, E.; Rotilio, G. Loss of GSH, oxidative stress, and decrease of intracellular $\mathrm{pH}$ as sequential steps in viral infection. J. Biol. Chem. 272:2700-2708; 1997.

[14] Lowry, O. H.; Rosebrough, N. J.; Farr, A. L.; Randall, R. J. Protein measurement with the Folin phenol reagent. J. Biol. Chem. 193:265-275; 1951.

[15] Ciriolo, M. R.; De Martino, A.; Lafavia, E.; Rossi, L.; Carri, M. T.; Rotilio, G. $\mathrm{Cu}, \mathrm{Zn}$-superoxide dismutase-dependent apoptosis induced by nitric oxide in neuronal cells. J. Biol. Chem. 275: 5065-5072; 2000.

[16] Ichijo, H.; Nishida, E.; Irie, K.; ten Dijke, P.; Saitoh, M.; Moriguchi, T.; Takagi, M.; Matsumoto, K.; Miyazono, K.; Gotoh, Y. Induction of apoptosis by ASK1, a mammalian MAPKKK that activates SAPK/JNK and p38 signaling pathways. Science 275:90-94; 1997.

[17] Wada, T.; Penninger, J. M. Mitogen-activated protein kinases in apoptosis regulation. Oncogene 23:2838-2849; 2004.

[18] Paolicchi, A.; Dominici, S.; Pieri, L.; Maellaro, E.; Pompella, A. Glutathione catabolism as a signaling mechanism. Biochem. Pharmacol. 64:1027-1035; 2002.

[19] Ding, W. Q.; Vaught, J. L.; Yamauchi, H.; Lind, S. E. Differential sensitivity of cancer cell to docosahexaenoic acid-induced cytotoxicity: the potential importance of down-regulation of superoxide dismutase 1 expression. Mol. Cancer Ther. 3:1109-1117; 2004.

[20] Pias, E. K.; Ekshyyan, O. Y.; Rhoads, C. A.; Fuseler, J.; Harrison, L.; Aw, T. Y. Differential effects of superoxide dismutase isoform expression on hydroperoxide-induced apoptosis in PC-12 cells. J. Biol. Chem. 278:13294-13301; 2003.

[21] Bode, A. M.; Dong, Z. Mitogen-activated protein kinase activation in UV-induced signal transduction. SCi. STKE 28:RE2 [DOI: 10.1126/ stke.2003.167.re2]; 2003.

[22] Cao, J.; Semenova, M. M.; Solovyan, V. T.; Han, J.; Coffey, E. T.; Courtney, M. J. Distinct requirements for p38alpha and c-Jun Nterminal kinase stress-activated protein kinases in different forms of apoptotic neuronal death. J. Biol. Chem. 279:35903-35913; 2004.

[23] Kuan, C. Y.; Yang, D. D.; Samanta Roy, D. R.; Davis, R. J.; Rakic, P.; Flavell, R. A. The Jnk1 and Jnk2 protein kinases are required for regional specific apoptosis during early brain development. Neuron 22:667-676; 1999.

[24] Herdegen, T.; Waetzig, V. AP-1 proteins in the adult brain: facts and fiction about effectors of neuroprotection and neurodegeneration. Oncogene 20:2424-2437; 2001.

[25] Filomeni, G.; Aquilano, K.; Rotilio, G.; Ciriolo, M. R. Reactive oxygen species-dependent c-Jun NH2-terminal kinase/c-Jun signalling cascade mediates neuroblastoma cell death induced by diallyl disulfide. Cancer Res. 63:5940-5949; 2003. 